Copyright (C 2014 IEEE. Personal use of this material is permitted. Permission from IEEE must be obtained for all other uses, in any current or future media, including reprinting/republishing this material for advertising or promotional purposes, creating new collective works, for resale or redistribution to servers or lists, or reuse of any copyrighted component of this work in other works. 


\section{Control, Operation and Power Sharing among Parallel Converter-Interfaced DERs in a Microgrid in the Presence of Unbalanced and Harmonic Loads}

\author{
Ruwan P.S. Chandrasena, Farhad Shahnia, Sumedha Rajakaruna \\ Department of Electrical \& Computer Engineering \\ Curtin University \\ Perth, Australia \\ ruwan.chandrasena@curtin.edu.au,farhad.shahnia@curtin.edu.au, s.rajakaruna@curtin.edu.au
}

\author{
Arindam Ghosh \\ Electrical and Computer Eng. School, \\ Queensland University of Technology \\ Brisbane, Australia \\ a.ghosh@qut.edu.au
}

\begin{abstract}
This paper demonstrates power management and control of DERs in an autonomous MG. The paper focuses on the control and performance of converter-interfaced DERs in voltage controlled mode. Several case studies are considered for a MG based on the different types of loads supplied by the MG (i.e. balanced three-phase, unbalanced, single-phase and harmonic loads). DERs are controlled by adjusting the voltage magnitude and angle in their converter output through droop control, in a decentralized concept. Based on this control method, DERs can successfully share the total demand of the MG in the presence of any type of loads. This includes proper total power sharing, unbalanced power sharing as well as harmonic power sharing, depending on the load types. The efficacy of the proposed power control, sharing and management among DERs in a microgrid is validated through extensive simulation studies using PSCAD/EMTDC.
\end{abstract}

Index Terms-Microgrid, DER, Voltage Control, Power Sharing.

\section{INTRODUCTION}

The ever increasing energy demand, along with the necessity of cost reduction and higher reliability requirements, are driving the modern power systems towards distributed generation (DG) as an alternative to the expansion of the current electricity distribution networks [1-2].

Microgrids (MG) are systems with clusters of DGs and loads. To deliver high quality and reliable power, the MG should appear as a single controllable unit that responds to changes in the system [3]. In MGs, parallel DGs are controlled to deliver the desired active and reactive power to the system while local signals are used as feedback to control the converters. The power sharing among the DGs can be achieved by controlling two independent quantities, frequency and fundamental voltage magnitude, at the converter output [4-7].

General introduction on MG basics, including the architecture, protection and power management is given in [8-9]. A review of ongoing research projects on MG in US, Canada, Europe and Japan is presented in [9-10]. Different power management strategies and controlling algorithms for a MG is proposed in [11-14]. Reference [15] has evaluated the feasibility of MGs operation during islanding and synchronisation periods.

One of the main issues still to be investigated in MGs is the effect of single-phase, unbalanced and harmonic loads on the control, operation and power sharing among parallel converter interfaced DERs. In the presence of balanced and linear loads, the converters can be operated in voltage control mode [16-19] or current control mode [20-23]. Majority of the researches for DER converter control in MGs utilise current control mode
[20-23]. For this, the output current reference for each converter should be calculated properly based on the load demand and the desired power sharing ratio among existing DERs. Later, using a proper switching mechanism in the converter, it can supply the MG by the desired reference current. This can be relatively complex when the MG is supplying single-phase, unbalanced or harmonic loads. In this case, the DERs not only should share the active and reactive power but also should share harmonic, negative and zero sequence currents. This needs a fast and accurate negative and zero sequence and harmonic current extraction mechanism as discussed in [24-25]. If the extracted sequence and harmonic currents mismatch the actual load requirement, the operation and power sharing of DERs can be significantly affected. However, by using a voltage control mode, there will be no necessity to measure and analyse the load current and to extract the sequence and harmonic current components.

In this paper, the operation, control and power sharing among parallel converter-interfaced micro sources, operating in voltage control mode, are investigated for grid-connected and autonomous modes. Through the extensive simulation results carried out by PSCAD/EMTDC, it is demonstrated that the DERs can successfully supply the MG loads with the desired power sharing ratios in the presence of single-phase, unbalanced and harmonic loads.

\section{MICROGRID STRUCTURE AND CONTROL}

Let us consider the MG system as shown in Fig. 1. The considered MG system consists of three converter-interfaced DERs. DERs such as photovoltaic cells (PV), fuel cells and batteries are usually connected to the MG through voltage source converters (VSC) and a properly tuned second order filter to the MG. The DERs are then controlled to supply the loads within the MG. It is to be noted that the considered DERs in this paper are working in voltage control mode and their output power, required by power sharing, are within their rated capabilities. Distribution Static Compensator (DSTATCOM) is installed at the secondary side of the distribution transformer to regulate the voltage at its Point of Common Coupling (PCC). The microgrid is maintained and operated using two control loops. The outer control loop satisfies proper power sharing among the DERs while the inner control loop is responsible of proper switching of DER converters in order to generate the desired voltage at their output. The DER and DSTATCOM converter structure in addition to outer and inner control mechanisms are discussed in detail below. 


\subsection{DER Converter Structure}

Let us assume that the DERs are connected to the MG through voltage source converters (VSC). The VSC structure consists of three single-phase H-bridges, using IGBTs, as shown in Fig. 2(a). Each IGBT has proper parallel reverse diode and snubber circuits. The outputs of each $\mathrm{H}$-bridge are connected to a single-phase transformer, with $1: a$ ratio, and three transformers are star-connected. In this figure, the resistance $R_{f}$ represents the switching and transformer losses, while the inductance $L_{f}$ represents the leakage reactance of the transformers and the filter capacitor $C_{f}$ is connected to the output of the transformers to bypass the switching harmonics.

\subsection{Outer Control Loop}

For a converter-interfaced DER, with the structure shown in Fig. 2(a), the instantaneous active and reactive power flows from converter output to its PCC are [16]

$$
\begin{aligned}
& p=\frac{\left|V_{T}\right| \times\left|V_{c f}\right|}{\omega L_{T}} \sin \left(\delta_{c f}-\delta_{T}\right) \\
& q=\frac{\left|V_{T}\right|}{\omega L_{T}}\left(\left|V_{c f}\right| \cos \left(\delta_{c f}-\delta_{T}\right)-\left|V_{T}\right|\right)
\end{aligned}
$$

where $V_{T}=\left|V_{T}\right| \angle \delta_{T}$ and $V_{c f}=\left|V_{c f}\right| \angle \delta_{c f}$ are respectively the RMS value of PCC voltage and AC filter capacitor voltage of DER. $L_{T}$ is the coupling inductance between PCC and inverter output which controls DER output power flow and $\omega$ is the angular frequency of the system. The switching frequency components in the instantaneous active power $(p)$ and reactive power $(q)$ can be filtered out to yield the average active power $(P)$ and reactive power $(Q)$ by passing them through a low pass filter. In [17], it was proposed that decentralized power sharing among the DERs in an autonomous MG can be achieved, similar to conventional droop control, by changing the voltage magnitude and angle of DERs as

$$
\begin{gathered}
\delta_{c f}=\delta_{\text {rated }}-m\left(P_{\text {rated }}-P\right) \\
V_{c f}=V_{\text {rated }}-n\left(Q_{\text {rated }}-Q\right)
\end{gathered}
$$

where $V_{\text {rated }}$ and $\delta_{\text {rated }}$ are respectively the rated voltage magnitude and angle of the DER when supplying the load with rated active and reactive power of $P_{\text {rated }}$ and $Q_{\text {rated }}$ while $m$ and $n$ are the droop coefficients.

The principle of decentralized power sharing in the MG is based on keeping the power output of DERs proportional to their ratings while the sum of the generated power from DERs is equal to the total required demand in the MG. This was mathematically simplified in [18] as

$$
\begin{array}{ll}
P_{\text {Load }}=\sum_{1}^{\text {DER number }} P_{i} & , \quad Q_{\text {Load }}=\sum_{1}^{\text {DER number }} Q_{i} \\
\frac{P_{i}}{P_{j}} \approx \frac{m_{j}}{m_{i}}=\frac{P_{i, \text { rated }}}{P_{j, \text { rated }}} & , \quad \frac{Q_{i}}{Q_{j}} \approx \frac{n_{j}}{n_{i}}=\frac{Q_{i, \text { rated }}}{Q_{j, \text { rated }}}
\end{array}
$$

where $i$ and $j$ show the number of each DER.

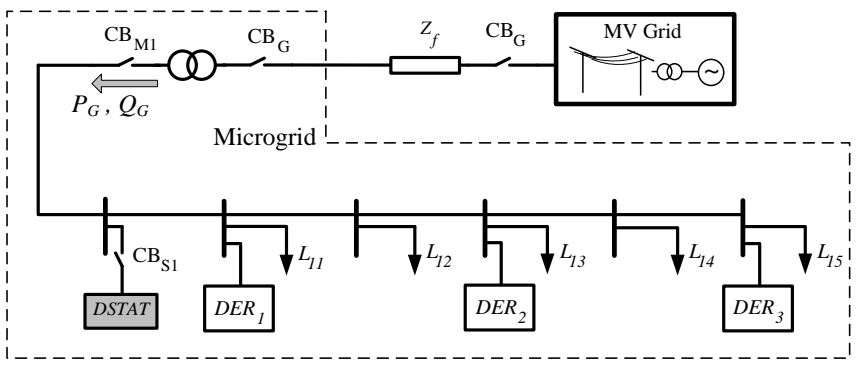

Fig. 1. Schematic diagram of the network under consideration.

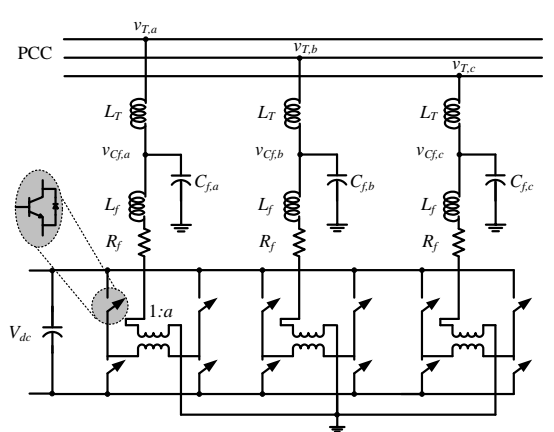

(a)

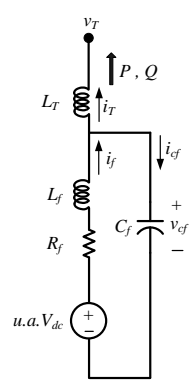

(b)
Fig. 2. (a) Schematic diagram of VSC for DERs, (b) Single-phase equivalent circuit of VSC.

\subsection{Inner Control Loop}

In this paper, the main concept of DER converter control is based on fixing the voltage magnitude and angle across $C_{f}$ as defined by the droop control from (2)-(3). This is achieved by appropriate switching of IGBTs in the converter. For this, let us consider the single-phase equivalent circuit of VSC as shown in Fig. 2(b). In this figure, $u \cdot a \cdot V_{d c}$ represents the converter output voltage, where $u$ is the switching function that can take on \pm 1 value depending on which pair of the IGBTs is turned on. The VSC is utilizing a closed-loop optimal robust controller based on state feedback to generate $u$. For this, two different state vectors can be assumed.

First, let us assume the state vector be defined as

$x=\left[\begin{array}{ll}v_{c f} & \tilde{i}_{f}\end{array}\right]^{T}$

where $v_{c f}$ represent the instantaneous voltage and $\tilde{i}_{f}$ is the high frequencies of $i_{f}$. Now, the reference vector, $x_{r e f}$ is to be calculated for each state variable, for each DER as

$$
\begin{array}{lll}
v_{c f, r e f, A}=V \angle \delta & , & \tilde{i}_{f, r e f, A}=0 \\
v_{c f, r e f, B}=V \angle \delta-120^{\circ} & , & \tilde{i}_{f, r e f, B}=0 \\
v_{c f, r e f, C}=V \angle \delta+120^{\circ} & , & \tilde{i}_{f, r e f, C}=0
\end{array}
$$

For DER control in this paper, $v_{c f, r e f}$ is calculated from (1) when the MG is in grid-connected mode and from (2) when in autonomous mode. From the circuit of Fig. 2(b), system state space description can be given as

$$
\dot{x}=A x+B_{1} u_{c}+B_{2} v_{T}
$$

where $u_{c}$ is the continuous time version of switching function $u$ and $v_{T}$ is assumed as load disturbance induced to the converter and neglected here. Discrete-time equivalent of (6) is 
$x(k+1)=F x(k)+G_{1} u_{c}(k)+G_{2} v_{T}(k)$

In (7), $u_{c}(k)$ is computed using a suitable state feedback control law. For this, switching control laws are given by

$$
u_{c}(k)=-K\left[x(k)-x_{r e f}(k)\right]
$$

where $K$ is a gain matrix. The gain matrix is obtained from Linear Quadratic Regulator (LQR) method which ensures the desired results for the system while the variations of system load and source parameters are within acceptable limits of reality. From $u_{c}(k)$, the switching function is then generated based on the error level as

$$
\begin{array}{llll}
\text { If } & u_{c}>+h & \text { then } & u=+1 \\
\text { elseif } & u_{c}<-h & \text { then } & u=-1
\end{array}
$$

where $h$ shows the error level and has a very small positive value. More detail on converter control is given in [26].

\subsection{Voltage Control in Autonomous Mode}

Based on the DER converter control described above, there is no direct voltage control in the MG in autonomous mode. This can be achieved if one of the DERs in MG regulates the network voltage. However, the DERs in residential networks can be owned by customers and are not responsible for network voltage support. Utilizing the converter of a DER to generate reactive power to support the network voltage profile will reduce the active power generation capacity of the converter. This will not be desired by their owners.

Alternatively, voltage regulation in a MG can be achieved using a DSTATCOM. This was presented and discussed in [27] and is not described here.

\section{STUDY CASES AND SIMULATION RESULTS}

For investigating the performance of the MG with several converter-interfaced DERs, different simulation cases are considered. The simulation cases are built to demonstrate the efficacy of the proposed voltage control mode for all DERs in proper power sharing among them in the network. The MG performance and power sharing among DERs are studied for different types of loads in the network such as balanced, unbalanced and harmonic. The DER, converter, filter, network and load parameters are given in the Appendix B.

\section{1. $M G$ with balanced loads}

Let us consider the simple structure of Fig. 1 to investigate the MG operation during grid-connected and autonomous modes. In grid-connected mode, each DER will generate its rated power and the extra load demand will be supplied by the grid or the extra generation will flow back into the grid. In autonomous mode, total power demand is shared among the DERs proportional to their rating.

First let us assume in the system of Fig. $1, \mathrm{CB}_{\mathrm{G}}, \mathrm{CB}_{\mathrm{M} 1}$ and $\mathrm{CB}_{\mathrm{S} 1}$ are closed. The system is assumed to be in steady state condition at $t=0 \mathrm{~s}$ and all the DERs are running in their rated condition. (a)

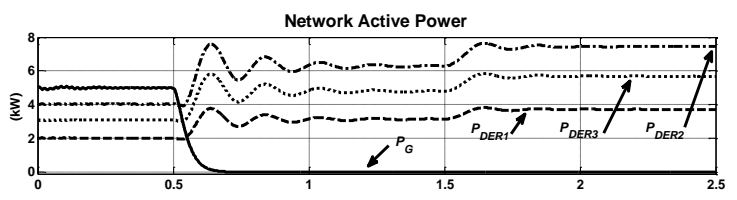

Single-Phase Active Power of DER-1
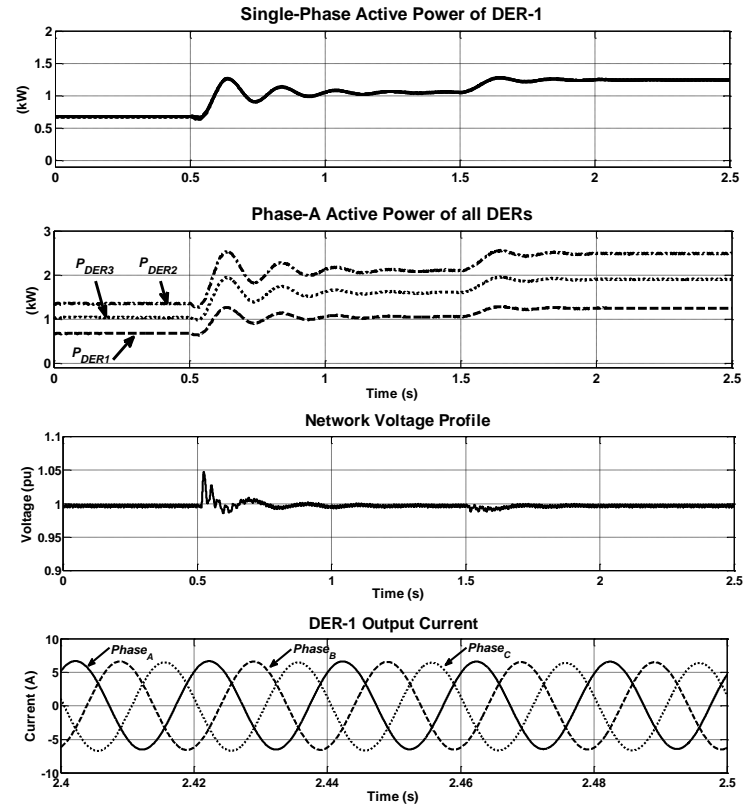

Fig. 3. Simulation results of MG in case 4.1:

(a) Active power dispatch of grid and 3 DERs,

(b) Single-phase active power output of DER-1,

(c) Active power output of all DERs in their phase-A,

(d) Network voltage profile in secondary side of transformer,

(e) Three-phase instantaneous current output of a DER-1.

At $t=0.5 \mathrm{~s}, \mathrm{CB}_{\mathrm{G}}$ opens and the grid is disconnected hence MG will work in autonomous mode. Therefore, the DERs have to increase their output power to satisfy the load demand within the MG. At $t=1.5 \mathrm{~s}$ one of the existing loads in the network is increased by $3 \mathrm{~kW}$. It can be seen that in both cases all DERs are sharing the load change proportional to their ratings.

Fig. 3(a) shows the active power dispatch of grid and $3 \mathrm{ex}-$ isting DERs in the MG between 0 and 2.5 seconds in the above-mentioned network. The single-phase active power output of one of the DERs (e.g. DER-1) is shown in Fig. 3(b). This is the same for all phases of A, B and C. From this figure, it can be seen that the network loads are three-phase balanced. The active power output in phase-A of all three DERs are also shown in Fig. 3(c). From this figure, it can be seen that all DERs are sharing the loads according to their ratios and they have an equal amount of power generated in each phase.

The voltage profile of the network is also shown in Fig. 3(d). As it can be seen in this figure, the network voltage is successfully regulated to 1 pu at all times.

The three-phase instantaneous current output of a sample DER (e.g. DER-1), shown in Fig. 3(e), confirms the DERs are generating balanced currents in their output.

\section{2. $M G$ with unbalanced loads}

In this case, let us assume in the system of Fig. 1, at $t=0 \mathrm{~s}$ $\mathrm{CB}_{\mathrm{G}}, \mathrm{CB}_{\mathrm{M} 1}$ and $\mathrm{CB}_{\mathrm{S} 1}$ are closed and the system is in steady state condition. At $t=0.5 \mathrm{~s}, \mathrm{CB}_{\mathrm{G}}$ is opened to disconnect the grid hence MG will work in autonomous mode. It is to be noted that distribution transformer is still connected to the $\mathrm{LV}$ side. 
(a)
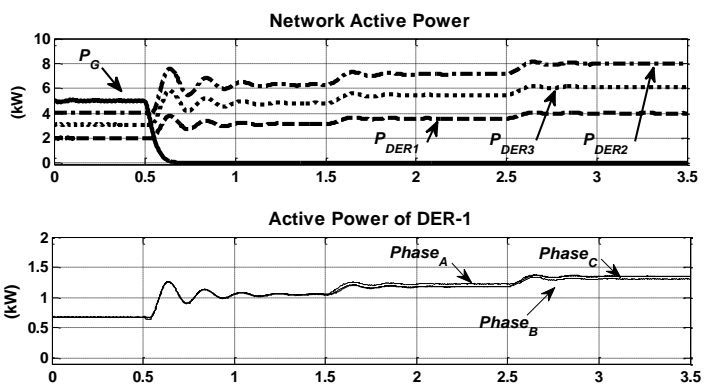

Phase-A Active Power of all DERs

(c)
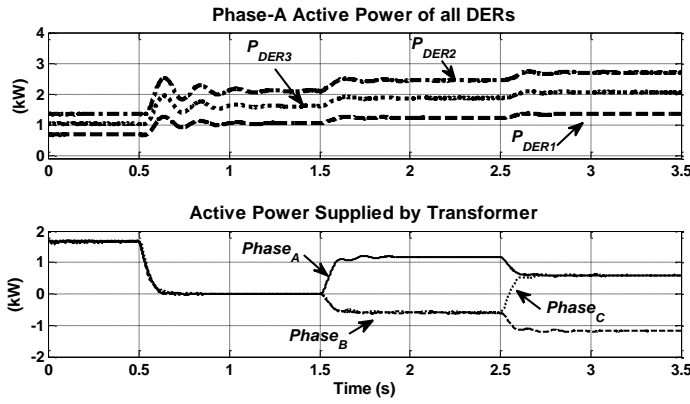

DER-1 Output Curren

(e)

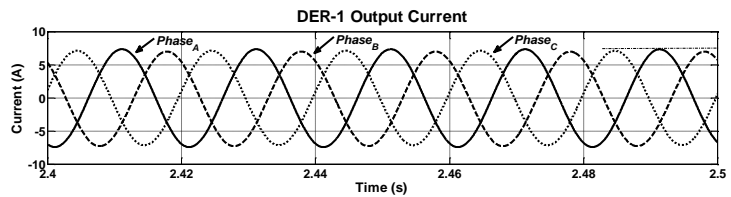

Fig. 4. Simulation results of MG in case 4.2

(a) Active power dispatch of grid and 3 DERs,

(b) Active power output of DER-1,

(c) Active power output of all DERs in their phase-A,

(d) Active power supplied by the distribution transformer,

(e) Three-phase instantaneous current output of a DER-1.

At $t=1.5 \mathrm{~s}$ a new single-phase $2 \mathrm{~kW}$ load is connected to phase-A. Later, at $t=2.5 \mathrm{~s}$ another single-phase $2 \mathrm{~kW}$ load is connected to phase-C.

Fig. 4(a) shows the active power dispatch of grid and 3 existing DERs in the MG between 0 and 3.5 seconds in the above-mentioned network. From this figure, it can be seen that, the total amount of active power generation among DERs are kept based on the desired power sharing ratio among them. The active power output of one of the DERs (e.g. DER-1) is shown in Fig. 4(b) for each phase individually. From this figure, it can be seen that for $t<1.5 \mathrm{~s}$, all three phases of DER-1 have an equivalent amount of generated active power. However, at $t=1.5 \mathrm{~s}$ when a single-phase load is connected to phaseA, the active power output of all phases of the DER increases. This increase is slightly higher for phase-A. This slight difference is further discussed in Appendix A. In a similar way, at $\mathrm{t}$ $=2.5 \mathrm{~s}$ when another single-phase load is connected to phase$\mathrm{C}$, the output active power of all phases of the DER increases but this increase is more for phase-C. Similar results are monitored in all the DERs of the MG. From this figure it can be seen that single phase load power demand is shared among the three phases of the DERs.

Fig. 4(c) shows the active power output of all DERs in their phase-A for the studied case. From this figure, it can be seen that for a load change in the network, the contribution level of each phase of the DERs in power generation is also based on the desired sharing ratio among them.

It is to be noted that, all phases of the DERs contribute to a single-phase (or unbalanced) load change in the network since there is a possibility of power circulation from one phase of MG to the other two phases through the windings of the distri- (a)
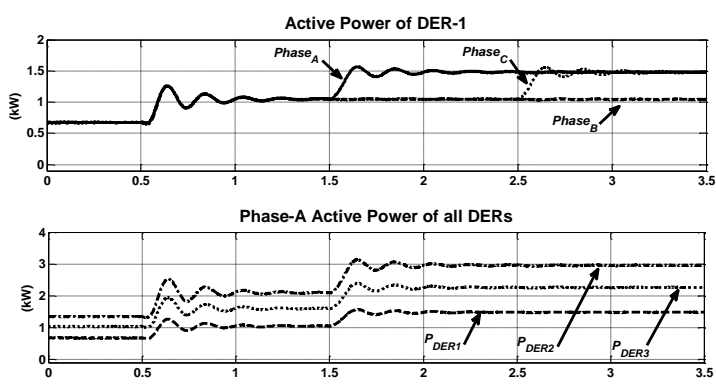

(c)
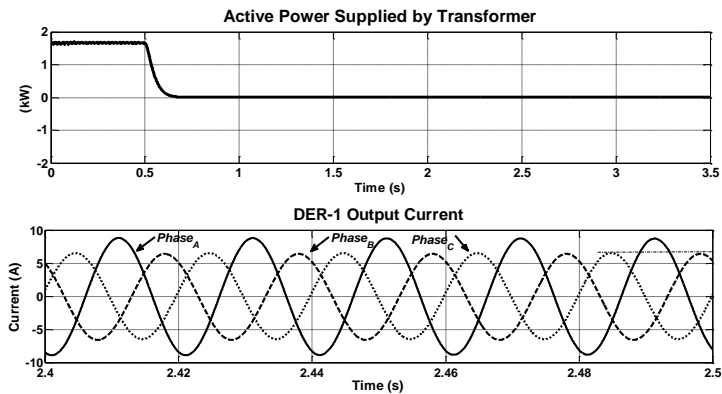

Fig. 5. Simulation results of MG in case 4.3:

(a) Active power output of DER-1,

(b) Active power output of all DERs in their phase-A,

(c) Active power supplied by the distribution transformer,

(d) Three-phase instantaneous current output of a DER-1.

bution transformer. Fig. 4(d) shows the active power supplied by the distribution transformer in the studied case. From this figure, it can be seen that the distribution transformer output power was zero when the MG was working in autonomous mode with balanced loads (i.e $0.5<t<1.5 \mathrm{~s}$ ). However, it can be seen that for $1.5<t<2.5 \mathrm{~s}$ there is a negative active power flow into the distribution transformer in phase- $\mathrm{B}$ and $\mathrm{C}$ which is circulated and retuned to phase $-\mathrm{A}$ where the single phase load is connected to. In a similar case, for $2.5<t<3.5 \mathrm{~s}$ there is a negative active power flow into the distribution transformer in phase- $\mathrm{B}$ which is circulated and retuned to phase- $\mathrm{A}$ and $\mathrm{C}$ where the single phase loads are connected to.

The three-phase instantaneous current output of a sample DER (e.g. DER-1) is shown in Fig. 4(e) which shows the DERs are generating unbalanced current in their output.

\subsection{MG with unbalanced loads and isolated transformer}

Now, let us consider the network of case 4.2 where the distribution transformer is isolated from the LV side when the grid is disconnected. Therefore, let us assume that at $t=0.5 \mathrm{~s}$, $\mathrm{CB}_{\mathrm{G}}$ and $\mathrm{CB}_{\mathrm{M} 1}$ open and $\mathrm{MG}$ works in autonomous mode.

As the distribution transformer is isolated from the LV side, no power circulation can happen among the three phases in the autonomous mode. Therefore, it is expected that by applying a single-phase (unbalanced) load change in the network, the DERs will contribute only in the phase in which the singlephase load is applied.

The active power output of one of the DERs (e.g. DER-1) is shown in Fig. 5(a) for each phase individually. From this figure, it can be seen that for $t<1.5 \mathrm{~s}$, all three phases of DER-1 have an equivalent amount of generated active power. Howev$\mathrm{er}$, at $t=1.5 \mathrm{~s}$ when a single-phase load is connected to phaseA, only the active power output of phase-A increases. In a similar way, at $\mathrm{t}=2.5 \mathrm{~s}$ when another single-phase load is connected to phase- $\mathrm{C}$, only the output active power of phase-C increases. Similar results are monitored in all the DERs of the MG. From this figure, it can be seen that single phase load 
power demand is only supplied by the relevant single-phase of the DERs.

Fig. 5(b) shows the active power output of all DERs in their phase-A for the studied case. From this figure, it can be seen that for a load change in the network, the contribution level of each phase of the DERs in power generation is based on the desired sharing ratio among them.

Fig. 5(c) shows the active power supplied by the distribution transformer in the studied case. As the transformer is isolated at $\mathrm{t}=0.5 \mathrm{~s}$, then its output power is zero.

The three-phase instantaneous current output of a sample DER (e.g. DER-1) is shown in Fig. 5(d) which shows the DERs are generating unbalanced current in their output.

\subsection{MG with harmonic loads}

In this section, let us consider the MG of Fig. 1. Let us assume that the system is in steady state condition at $t=0 \mathrm{~s}$ and at $t=0.5 \mathrm{~s}$, the grid is disconnected (i.e. $\mathrm{CB}_{\mathrm{G}}$ is opened) and MG will work in autonomous mode.

At $t=1.5 \mathrm{~s}$ a new three-phase harmonic load of $3 \mathrm{~kW}$ is connected to the network. At $t=2.5 \mathrm{~s}$, its demand is increased to $6 \mathrm{~kW}$. The harmonic load has a Total Harmonic Distortion (THD) of $25.5 \%$ with the Fast Fourier Transform (FFT) Spectrum as shown in Fig. 6(a).

Fig. 6(b) shows the active power dispatch of grid and 3 existing DERs in the MG between 0 and 3.5 seconds in the above-mentioned network. The single-phase active power output of one of the DERs (e.g. DER-1) is shown in Fig. 6(c). This is the same for all phases of A, B and C. The active power output in phase-A of all three DERs are also shown in Fig. $6(d)$. From these figures, it can be seen that as the load is supplied from the three-phase network, its demand is shared equally among the three phases of each DER. However, the DERs share the extra demand based on their desired power sharing ratio.

The three-phase instantaneous current output of a sample DER (e.g. DER-1) is shown in Fig. 6(e) which shows the output current of the DERs are distorted as required by the network harmonic load.

\section{CONCLUSION}

The power management and control of converter-interfaced DERs were discussed in this paper, for autonomous operation of a MG with balanced, unbalanced and harmonic loads. In many MG related researches, the converter-interfaced micro sources are current controlled. In such a case, if the MG supplies unbalanced loads, detailed calculations are required to extract the required positive, negative and zero sequences of the reference current components for the DERs. Similarly, if the MG supplies nonlinear and harmonic loads, detailed calculations are required to extract the required different harmonic components for the current reference for the DERs. These calculations are later utilized for proper total power sharing, unbalanced power sharing and harmonic power sharing among DERs in the MG. However, if the converters are voltage controlled, there is no necessity for these complicated current reference extractions and calculations. In this paper, through several case studies, it was verified that proper total power sharing, harmonic power sharing and unbalance power sharing among DERs can be achieved through voltage controlled converters in the MG without any complicated current reference extractions for DERs. (a)

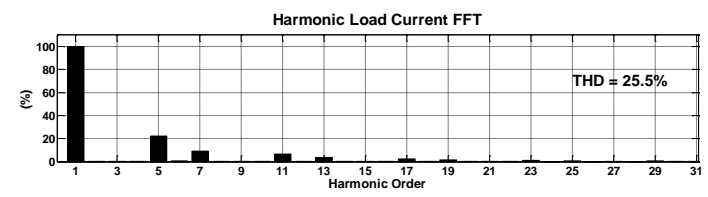

(b)
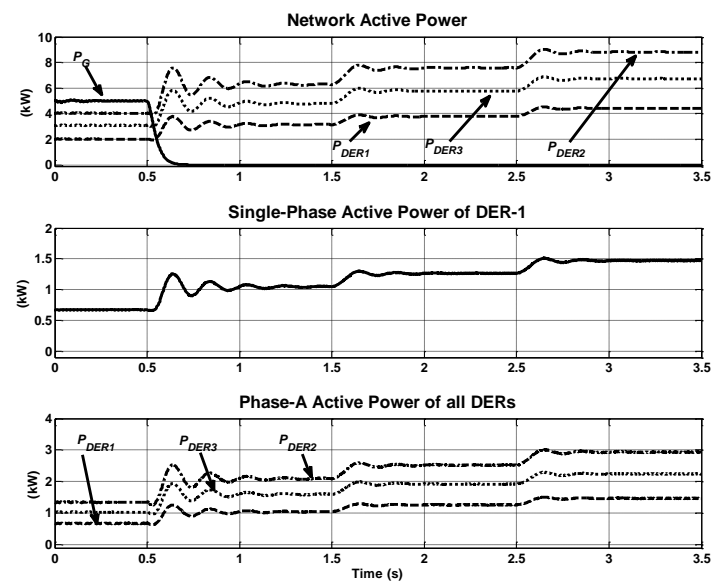

(e)

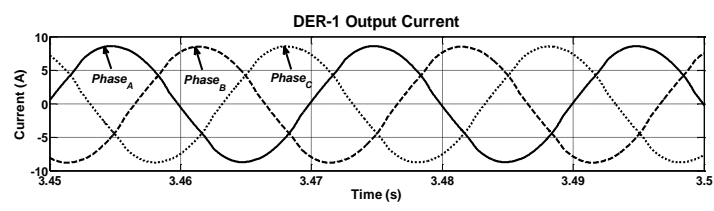

Fig. 6. Simulation results of MG in case 4.4:

(a) FFT Spectrum of harmonic load current,

(b) Active power dispatch of grid and 3 DERs,

(c) Single-phase active power output of DER-1,

(d) Active power output of all DERs in their phase-A,

(e) Three-phase instantaneous current output of a DER-1.

\section{APPENDIX A}

\section{Power Circulation through Transformer}

Let us consider the simple network illustrated in Fig. 7. A three-phase converter-interfaced DER in voltage control mode is connected to Bus-3 to supply a single-phase load in Bus-2 connected to phase-A. A three-phase DSTATCOM is installed in Bus-1 to regulate its PCC to a three-phase balanced voltage of $1 \mathrm{pu}$. Only the downstream of $\Delta / \mathrm{Y}-$ Grounded transformer is connected while its upstream side is open-circuited. The $\Delta / Y_{-}$ Grounded transformer can circulate the reverse fed current and power into one of its $\mathrm{Y}$-grounded windings into its two other Y-Grounded windings due to its primary delta configuration.

First, let us assume that CB-1 is open and the DSTATCOM is not connected. In this case, the single-phase load will be supplied by $i_{a 1}$ and $i_{a 2}$ as

$$
\begin{aligned}
& i_{a 1}=i_{g a}=\frac{v_{g a}-v_{\text {load }}}{\alpha Z} \\
& i_{a 2}=i_{g b}+i_{g c}=\frac{v_{g b}-v_{\text {load }}}{Z+Z_{\text {tr_eq }}+(1-\alpha) Z}+\frac{v_{g c}-v_{\text {load }}}{Z+Z_{t r_{-} e q}+(1-\alpha) Z}
\end{aligned}
$$

where $i_{a l}$ is the phase-A current supplied from the generator side, $i_{a 2}$ is the phase-A current supplied from the transformer side, $v_{g a}, v_{g b}$ and $v_{g c}$ are the three-phase output voltage of the DER, $i_{g a}, i_{g b}$ and $i_{g c}$ are the three-phase current output of the DER, $v_{\text {load }}$ is the voltage at load PCC, $Z$ is the feeder impedance between the buses, $0<\alpha<1$ and $Z_{t r, e q}$ is the equivalent impedance between two terminals of the transformer. From Fig. (7), we have

$\alpha Z<\left(Z+Z_{t r_{-} e q}+(1-\alpha) Z\right)$ 


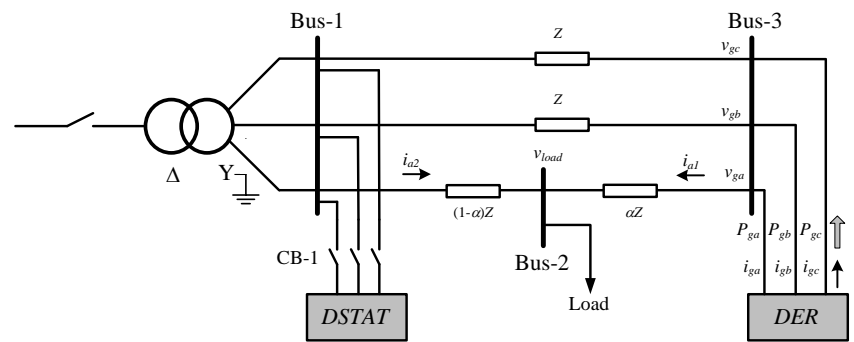

Fig. 7. Simplified network under consideration.

Therefore, it is expected that $i_{a 1}>i_{a 2}$. Hence,

$i_{g a}>i_{g b}=i_{g c}$ or $\quad P_{g a}>P_{g b}=P_{g c}$

From (10), it can be seen that the amount of current (or power) supplied from DER in its phase-B and $\mathrm{C}$ are highly dependent on the equivalent impedance of the transformer and the line.

Now, let us assume that CB-1 is closed and the DSTAT$\mathrm{COM}$ is regulating its PCC to 1 pu balanced voltage. In this case, $i_{a 1}$ will be same as in (10) while $i_{a 2}$ will be

$i_{a 2}=\frac{1 \angle \delta-v_{\text {load }}}{(1-\alpha) Z}$

where $\delta$ is the angle of phase-A in Bus-1. Therefore, from (10) and (13), it can be seen that there will be always a small difference between $i_{a 1}$ and $i_{a 2}$. The same difference will be reflected in $i_{g a}, i_{g b}$ and $i_{g c}$ in the output of the DER. Hence, it is expected that there will be a slight difference between $P_{g a}$ compared to $P_{g b}$ and $P_{g c}$. This difference is dependent on the distance between load and DER and transformer as well as the load demand.

\section{APPENDIX B}

Table I. Technical data of the network parameters of Fig. 1.

\begin{tabular}{ll}
\hline MV Network & $11 \mathrm{kV} \mathrm{L}-\mathrm{L}$ RMS, $50 \mathrm{~Hz}$ \\
MV Line Impedance & $R=0.2 \Omega, L=10 \mathrm{mH}$ \\
LV Feeder & $415 \mathrm{~V} \mathrm{~L}-\mathrm{L}$ RMS, $50 \mathrm{~Hz}$ \\
LV Line Impedance & $R=0.02 \Omega, L=1 \mathrm{mH}$ \\
Transformer & $30 \mathrm{kVA}, 11 \mathrm{kV} / 415 \mathrm{~V}$, Three-Phase, $50 \mathrm{~Hz}$, \\
& $\Delta / \mathrm{Y}-\mathrm{Grounded}, Z_{I}=5 \%$ \\
Balanced Three Phase Loads & $P=2.7 \mathrm{~kW}, P F=0.95$ \\
Single Phase Load & $P=2 \mathrm{~kW}, P F=0.95$ in Phase A \\
Single Phase Load & $P=4 \mathrm{~kW}, P F=0.95 \mathrm{in}$ Phase B \\
DER VSCs and Filters & $R_{f}=0.1 \Omega, L_{f}=0.36 \mathrm{mH}, C_{f}=50 \mu \mathrm{F}$, \\
& $V_{d c}=150 \mathrm{~V}, a=3.33, h=10^{-5}$ \\
DSTATCOM VSC and & $R_{f}=1 \mathrm{~m} \Omega, L_{f}=4 \mathrm{mH}, C_{f}=25 \mu \mathrm{F}, L_{T}=10$ \\
Filter & $\mathrm{mH}, V_{d c}=1 \mathrm{kV}, \mathrm{C}_{\mathrm{dc}}=2000 \mu \mathrm{F}, a=1, h=10^{-5}$ \\
\hline
\end{tabular}

Table II. Technical data of DERs and droop control coefficients in Fig. 1.

\begin{tabular}{lcccc}
\hline DER & $\begin{array}{c}\text { DER Rating } \\
{[\mathrm{kW}]}\end{array}$ & $\begin{array}{c}\text { Coupling Inductance } \\
\left(L_{T}\right)[\mathrm{mH}]\end{array}$ & $\begin{array}{c}m \\
{[\mathrm{rad} / \mathrm{kW}]}\end{array}$ & $\begin{array}{c}n \\
{[\mathrm{~V} / \mathrm{kVAr}]}\end{array}$ \\
DER-1 & 2.0 & 76.2 & 3.14 & 1.8 \\
DER-2 & 4.0 & 38.1 & 1.57 & 0.9 \\
DER-3 & 3.06 & 49.8 & 2.05 & 1.17 \\
\hline
\end{tabular}

\section{REFERENCES}

[1] B Kroposki, C. Pink, R. DeBlasio, H. Thomas, M. Simões and P.K. Sen, "Benefits of Power Electronic Interfaces for Distributed Energy Systems," IEEE Trans. on Energy Conversion, vol. 25, no. 3, pp. 901-908, 2010 .

[2] T. Senjyu, T. Nakaji, K. Uezato, and T. Funabashi, "A hybrid power system using alternative energy facilities in isolated island," IEEE Trans. on Energy Conversion., vol. 20, no. 2, pp. 406-414, 2005.

[3] R.H. Lasseter, "MicroGrids," IEEE Power Engineering Society Winter Meeting, vol. 1, pp. 305-308, 2002.

[4] A.G. Madureira and J.A. Pecas Lopes, "Coordinated Voltage Support in Distribution Networks with Distributed Generation and Microgrids," IET Renewable Power Generation, vol. 3, no. 4, pp. 439-454, 2009.
[5] K. Pandiaraj, P. Taylor, N. Jenkins, and C. Robb, "Distributed Load Control of Autonomous Renewable Energy Systems," IEEE Trans. on Energy Conversion, vol. 16, no. 1, pp. 14-19, 2001.

[6] R. Majumder, G. Ledwich, A. Ghosh, S. Chakrabarti and F. Zare, "Droop Control of Converter-Interfaced Microsources in Rural Distributed Generation," IEEE Trans. on Power Delivery, vol. 25, no. 4, pp. 2768-2778, 2010

[7] S.J. Ahn, J.W. Park, I.Y. Chung, S.I. Moon, S.H. Kang and S.R. Nam, "Power-Sharing Method of Multiple Distributed Generators Considering Control Modes and Configurations of a Microgrid," IEEE Trans. Power Delivery, vol. 25, no. 3, pp. 2007-2016, 2010.

[8] B. Kroposki, R. Lasseter, T. Ise, S. Morozumi, S. Papatlianassiou and N. Hatziargyriou, "Making microgrids work," IEEE Power Energy Magazine, vol. 6, no. 3, pp. 40-53, 2008.

[9] F. Katiraei, R. Iravani, N. Hatziargyriou and A. Dimeas, "Microgrids management," IEEE Power Energy Magazine, vol. 6, no. 3, pp. 54-65, 2008 .

[10] M. Barnes, J. Kondoh, H. Asano, J. Oyarzabal, G. Ventakaramanan, R. Lasseter, N. Hatziargyriou and T. Green, "Real-World Micro Grids-An Overview," IEEE Int. Conf. on Systems Engineering, pp. 1-8, 2007.

[11] I.Y. Chung, W. Liu, D.A. Cartes, E.G. Collins and S.I. Moon, "Control Methods of Inverter-Interfaced Distributed Generators in a Microgrid System," IEEE Trans. on Industrial Applications, vol. 46, no. 3, pp. 1078-1088, 2010.

[12] M. Dewadasa, R. Majumder, A. Ghosh and G. Ledwich, "Control and Protection of a Microgrid with Converter Interfaced Micro Sources," IEEE Int. Conf. on Power Systems, pp. 1-6, 2009.

[13] R. Majumder, A. Ghosh, G. Ledwich and F. Zare, "Power Sharing and Stability Enhancement of an Autonomous Microgrid with Inertial and Non-Inertial DGs with DSTATCOM," Int. Conf. on Power Systems (ICPS), pp. 1-6, 2009

[14] F. Blaabjerg, R. Teodorescu, M. Liserre and A.V. Timbus, "Overview of Control and Grid Synchronization for Distributed Power Generation Systems," IEEE Trans. on Industrial Electronics, vol. 53, no. 5, pp. 1398-1409, Oct. 2006.

[15] C.L. Chen, Y. Wang, J.S. Lai, Y.S. Lee and D. Martin, "Design of Parallel Inverters for Smooth Mode Transfer Microgrid Applications," IEEE Trans. on Power Electronics, vol. 25, no. 1, pp. 6-15, 2010.

[16] F. Shahnia, R. Majumder, A. Ghosh, G. Ledwich and F. Zare, "Voltage imbalance analysis in residential low voltage distribution networks with rooftop PVs," Electric Power Systems Research, vol. 81, Issue 9, pp. 1805-1814, Sep. 2011.

[17] R. Majumder, A. Ghosh, G. Ledwich and F. Zare, "Angle Droop Versus Frequency Droop in a Voltage Source Converter Based Autonomous Microgrid," IEEE PES Society General Meeting, pp. 1-8, 2009.

[18] F. Shahnia, R. Majumder, A. Ghosh, G. Ledwich and F. Zare, "Operation and Control of a Hybrid Microgrid Containing Unbalanced and Nonlinear Loads," Electric Power Systems Research, vol. 80, no. 8, pp 954-965, Aug. 2010.

[19] R. Majumder, F. Shahnia, A. Ghosh, G. Ledwich, M. Wishart and F Zare, "Operation and Control of a Microgrid Containing Inertial and Non-inertial Micro Sources," IEEE Region 10 Conf., 2009.

[20] D. De and V. Ramanarayanan, "Decentralized Parallel Operation of Inverters Sharing Unbalanced and Nonlinear Loads," IEEE Trans. on Power Electronics, vol. 25, no. 12, pp. 3015-3025, Dec. 2010.

[21] U. Borup, F. Blaabjerg, and P. N. Enjeti, "Sharing of nonlinear load in parallel-connected three-phase converters," IEEE Trans. on Industrial Application, vol. 37, no. 6, pp. 1817-1823, Dec. 2001.

[22] M. Hamzeh, H. Karimi, and H. Mokhtari, "A New Control Strategy for a Multi-Bus MV Microgrid Under Unbalanced Conditions," IEEE Trans. on Power Systems, vol. 27, no. 4, pp. 2225-2232, Nov. 2012.

[23] T. Vandoorn, B. Meersman, J. De Kooning, and L. Vandevelde, "Controllable Harmonic Current Sharing in Islanded Microgrids: DG Units With Programmable Resistive Behavior Toward Harmonics," IEEE Trans. on Power Delivery, vol. 27, no. 2, pp. 831-841, Apr. 2012.

[24] U. Borup, F. Blaabjerg and P.N. Enjeti, "Sharing of Nonlinear Load in Parallel-Connected Three-Phase Converters," IEEE Trans. on Industrial Applications, vol. 37, no. 6, pp. 1817-1823, 2001

[25] C. Sao and P.W. Lehn, "Voltage Balancing of Converter Fed Microgrids with Single Phase Loads," IEEE Power Energy Society General Meeting, pp. 1-7, 2008.

[26] A. Ghosh and G. Ledwich, Power Quality Enhancement using Custom Power Devices, Kluwer Academic Publishers, 2002.

[27] F. Shahnia, R.P.S. Chandrasena, S. Rajakaruna and A. Ghosh, "Autonomous Operation of Multiple Interconnected Microgrids with SelfHealing Capability," IEEE Power Energy Society General Meeting, pp. $1-5,2013$. 